
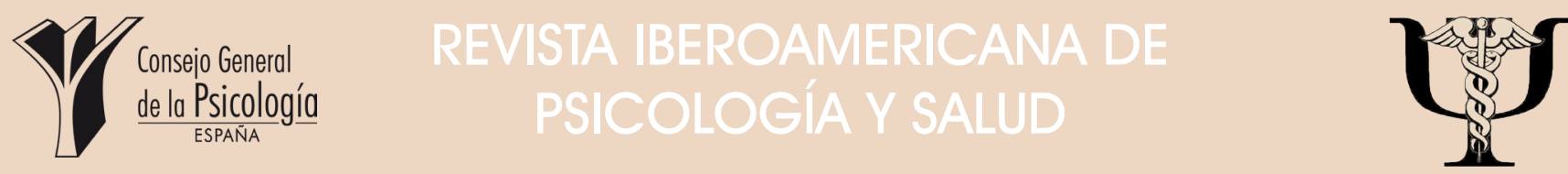

Revista Oficial de la Federación Iberoamericana de Asociaciones de Psicología (FIAP)

[Official Journal of the Latin-American Federation of Psychological Associations]

\title{
Predictores del cambio clínicamente significativo en una intervención de prevención de la depresión.
}

\author{
Vanessa Blanco ${ }^{*}$, Patricia Otero², Lara López ${ }^{3}$, Ángela Torres ${ }^{4}$ y Fernando L. Vázquez ${ }^{3}$ \\ 'Departamento de Psicología Evolutiva y de la Educación, Universidad de Santiago de Compostela, Santiago de Compostela, España. \\ ${ }^{2}$ Departamento de Psicología, Universidad de A Coruña, A Coruña, España. \\ ${ }^{3}$ Departamento de Psicología Clínica y Psicobiología, Universidad de Santiago de Compostela, España. \\ ${ }^{4}$ Departamento de Psiquiatría, Radiología y Salud Pública, Universidad de Santiago de Compostela, España.
}

- Recibido: 28 - Abril - 2016 . Aceptado: 26 - Octubre - 2016

\section{RESUMEN}

Una de las limitaciones de la investigación sobre prevención indicada de la depresión es que pocos trabajos han analizado la significación clínica de los efectos de la intervención. En el presente estudio, se asignaron al azar 170 cuidadoras no profesionales con síntomas depresivos a un grupo de intervención cognitivo-conductual $(n=88)$ o a un grupo control de atención habitual $(n=82)$. La intervención se aplicó en cinco sesiones de 90 minutos en grupos de aproximadamente cinco participantes, por psicólogos previamente entrenados. En la posintervención se encontró un porcentaje significativamente mayor de cuidadoras que alcanzaron un cambio clínicamente significativo en el grupo de intervención en comparación con el grupo control $\left(62.5 \%\right.$ vs. $15.9 \% ; x^{2}(1, N=170)=38.48, p<$ $.001)$. Esto se mantuvo hasta los 12 meses de seguimiento, donde el $81.8 \%$ del grupo de intervención obtuvo un cambio clínicamente significativo frente al $17.1 \%$ del grupo control $\left(x^{2}(1, N=170)=71.18, p<.001\right)$. Las variables predictoras del cambio clínico para el grupo de intervención en la posintervención fueron dedicarse a las tareas domésticas y una mayor edad de la persona cuidada. En el seguimiento a 12 meses no se encontró ninguna variable predictora.

PALABRAS CLAVE: Predictores, Depresión, Prevención, Significación clínica, Cuidadores.

\section{ABSTRACT}

Clinically significant predictors of change in an intervention for the prevention of depression. One of the limitations of research in indicated prevention of depression is that few works have analyzed the clinical significance of the effects of the interventions. In this study, 170 non-professional caregivers with depressive symptoms were randomized to a cognitive-behavioral intervention group ( $n=88$ ) or to a usual-care control group $(n=82)$. The intervention was implemented in five weekly 90-minute sessions in groups of approximately five participants by trained psychologists. At postreatment, there was a significantly higher percentage of caregivers who achieved a clinically significant change in the intervention group compared to the control group $\left(62.5 \%\right.$ vs. $\left.15.9 \% ; x^{2}(1, N=170)=38.48, p<.001\right)$. This was maintained until 12-month follow-up, when $81.8 \%$ of the intervention group obtained a clinically significant change compared to $17.1 \%$ in the control group $\left(x^{2}(1, N=170)=71.18, p<.001\right)$. The variables that predicted clinical change for the intervention group at postreatment were to be devoted to domestic work and older age of the care recipient. No predictors were found for the 12-month follow-up.

La depresión es uno de los trastornos mentales más frecuentes en la población de cuidadores no profesionales, con una

\footnotetext{
*Correspondencia: Vanessa Blanco.
}

Grupo de Investigación en Salud Mental y Psicopatología (GRISAMP). Facultad de Psicología. Universidad de Santiago de Compostela.

Rúa Xosé María Suárez Núñez, s/n, Campus Vida,

Código Postal: 15782, Santiago de Compostela, España.

E-mail:vanessa.blanco@usc.es prevalencia del $8.9 \%$ (Torres et al., 2015), lo que supone una cifra notablemente más elevada que la hallada en la población general española (Gabilondo et al., 2010). Este dato es especialmente preocupante si tenemos en cuenta que la depresión es un trastorno discapacitante que causa un gran sufrimiento para las personas y sus familias, perturba las actividades diarias de manera significativa, empeora el resultado de otros problemas de salud y es un factor de riesgo para el suicidio (Wang y Kessler, 2006). 
Por tanto, resulta crucial desarrollar programas de prevención eficaces. Dentro del campo de la prevención son especialmente relevantes las intervenciones de prevención dirigidas a aquellas personas que ya manifiestan síntomas pero que todavía no han desarrollado un episodio de depresión clínica (i.e., prevención indicada; Institute of Medicine, 1994), dado que tener elevados síntomas depresivos aumenta el riesgo para desarrollar trastornos depresivos (Cuijpers, Smit y Willemse, 2005).

Las intervenciones de prevención indicada de la depresión con mayor evidencia empírica son las cognitivo-conductuales (Vázquez, Blanco, Torres, Otero y Hermida, 2014), que mostraron eficacia en la reducción de los síntomas depresivos en diversos estudios (e.g., Konnert, Dobson y Stelmach, 2009; Otero, Smit et al., 2015; Stice, Rohde, Seeley y Gau, 2008; Vázquez et al., 2016). Sin embargo, una limitación de los estudios de prevención indicada de la depresión es que la mayoría utilizaron únicamente pruebas de significación estadística como base para valorar los efectos de las intervenciones; y el hecho de que una intervención tenga efectos favorables basándose en las medias no implica necesariamente que el cambio producido en los participantes alcance una significación clínica. Dicha significación clínica se refiere a las implicaciones prácticas del efecto de una intervención o la diferencia real que ésta produce en la vida cotidiana del paciente (Kazdin, 1999).

Hasta la actualidad, sólo siete estudios de prevención indicada de la depresión informaron de la significación clínica de las intervenciones (Konnert et al., 2009; Liu et al., 2009; Stice et al., 2008; Stice, Rohde, Gau y Wade, 2010; Vázquez et al., 2012, 2013; Vázquez, Hermida et al., 2014). En el estudio de Konnert et al. (2009), 43 personas con síntomas depresivos que vivían en una residencia de ancianos fueron asignadas aleatoriamente a un grupo de tratamiento cognitivo-conductual o a un grupo control de atención habitual, encontrando una recuperación clínicamente significativa del 95\% para el grupo de intervención a los 6 meses de seguimiento. En el estudio de Liu et al. (2009), 52 adultos con síntomas depresivos se asignaron al azar a un grupo de tratamiento de biblioterapia o un grupo control de lista de espera, hallando un $48 \%$ de sujetos clínicamente recuperados del grupo de tratamiento en la posintervención. En el estudio de Stice et al. (2008), 341 adolescentes en riesgo para desarrollar depresión fueron aleatorizados a una intervención cognitivo-conductual, grupos de apoyo expresivo, biblioterapia o un grupo control de atención. Se obtuvo un $43 \%$ de sujetos clínicamente recuperados en el grupo de terapia cognitivo-conductual en el seguimiento a los 6 meses, $36 \%$ en el grupo de apoyo expresivo, 35\% en el de biblioterapia y $20 \%$ en el grupo control. A los dos años de seguimiento (Stice et al., 2010), hubo un $31.5 \%$ de sujetos clínicamente recuperados en el grupo de terapia cognitivo-conductual, $25 \%$ en el de apoyo expresivo, $12.5 \%$ en el de biblioterapia y $17.9 \%$ en el grupo control. En el estudio de Vázquez et al. (2012), en una muestra de 133 estudiantes universitarios con síntomas depresivos elevados asignados aleatoriamente a un programa de entrenamiento en relajación o una intervención cognitivo-conductual, se encontró una recuperación clínicamente significativa del $39.7 \%$ y $62.9 \%$ respectivamente en la posintervención, y del $47.6 \%$ y $45.7 \%$ respectivamente en el seguimiento a 6 meses. En el estudio de Vázquez et al. (2013) se asignaron al azar 173 cuidadoras con síntomas depresivos al grupo de intervención en solución de problemas o un grupo control de atención habitual, y se encontró un $80.9 \%$ de cuidadoras clínicamente recuperadas en el grupo de intervención frente al 11.9\% del grupo control en la posintervención. Por último, en el estudio de Vázquez, Hermida et al. (2014) se evaluó la eficacia de la presente intervención cognitivo-conductual en comparación con un grupo control de atención habitual en una muestra de 170 cuidadoras, y se encontró una significación clínica en el $70.5 \%$ de cuidadoras del grupo de intervención frente al $24.4 \%$ del grupo control en la posintervención.

No obstante, estos estudios presentan dos importantes limitaciones. Una es que el análisis de la significación clínica del cambio terapéutico fue considerado como un resultado secundario. Otra es que en la mayoría de estos trabajos la significación clínica se analizó en base a un punto de corte determinado en una escala de síntomas depresivos. Sólo tres de estos estudios (Liu et al., 2009; Stice et al., 2008, 
2010) utilizaron el Índice de Cambio Fiable (ICF) propuesto por Jacobson y Truax (1991), el cual es un estimador del cambio clínicamente significativo que permite mensurar cuánto cambio ha ocurrido durante el transcurso de la terapia.

Además, hasta la fecha, la investigación sobre predictores en la prevención indicada es limitada, especialmente cuando el efecto de las intervenciones se valora en base a la significación clínica, sobre lo que no existe ningún estudio. Los predictores son variables que están presentes antes del tratamiento (Kraemer, Wilson, Fairburn y Agras, 2002) y que tienen un efecto sobre el resultado. Sólo algunos estudios previos de otros tipos de prevención y tratamiento de la depresión han estudiado las variables predictoras de los resultados de la intervención cognitivoconductual, encontrando como variables predictoras el sexo femenino (Spek, Nyklíček, Cuiipers y Pop, 2008), el estado civil (Dobkin et al., 2012) y un mayor nivel de sintomatología depresiva en la línea base (Dobkin et al., 2012; Spek et al., 2008).

El objetivo de este trabajo fue determinar la significación clínica y los predictores de este resultado de una intervención cognitivoconductual para la prevención de la depresión en una muestra de cuidadoras con síntomas depresivos elevados.

\section{MÉTODO}

El presente estudio representa un análisis secundario de un ensayo controlado aleatorizado publicado previamente en el que se comparó una intervención cognitivoconductual con un grupo control de atención habitual. La información metodológica ha sido descrita en otro estudio (Vázquez et al., 2016); aquí se destacan los aspectos más importantes del Método.

\section{- MUESTRA}

La muestra del estudio se obtuvo del registro oficial de cuidadores de la Comunidad Autónoma de Galicia que posee la Xunta de Galicia en España. Los participantes debían ser mujeres, cuidadoras principales de un familiar en situación de dependencia, con una puntuación de 16 o más en la Escala para la Depresión del Centro de Estudios Epidemiológicos (Center for Epidemiologic Studies Depression Scale [CES-D]; Radloff, 1977; versión española de Vázquez, Blanco y López, 2007), sin historia de episodios depresivos determinados por los criterios DSM-IV. Los criterios de exclusión fueron haber recibido tratamiento psiquiátrico o psicológico en los dos últimos meses, presentar otros trastornos del Eje I que pudieran actuar como variable de confusión (e.g., trastornos bipolares I y II, trastornos psicóticos), presentar condiciones psicológicas o médicas que requieran intervención inmediata (e.g. ideación suicida) o imposibiliten la realización del estudio (e.g. deficiencia mental, deterioro cognitivo significativo, deficiencia auditiva severa), participar en otro estudio, previsión de cambio de domicilio o institucionalización del familiar o pronóstico terminal del familiar cuidado.

De los 450 sujetos evaluados, 174 cumplieron los criterios de elegibilidad y de éstos 4 (2.3\%) declinaron la participación en el estudio por no tener a nadie que cuidara a su familiar dependiente en su ausencia, falta de tiempo, porque el horario de las intervenciones coincidía con su horario de trabajo o por falta de interés en la intervención. Quedó una muestra final de 170 cuidadoras, con una media de edad de 55.1 años (DT $=9.0$ ), las cuales fueron asignadas al azar por un experto en estadística ajeno al estudio mediante una tabla de números aleatorios a un grupo de intervención cognitivo-conductual $(n=88)$ o a un grupo control de atención habitual $(\mathrm{n}=82)$.

Para minimizar la pérdida de sujetos se siguieron algunas de las estrategias recomendadas por Grady, Cummings y Hulley (2007/2008) para este tipo de estudios. Entre otras, excluir probables pérdidas, hacer la intervención fácil, realizar las sesiones a una hora conveniente y obtener diversos números de contacto para poder realizar los seguimientos.

La investigación se realizó de acuerdo con la última versión de la Declaración de Helsinki y fue aprobada por el comité ético de la Universidad de Santiago de Compostela. Todos los participantes dieron su consentimiento informado. La participación fue completamente voluntaria, sin ninguna clase de incentivo económico o de otro tipo. 


\section{- MEDIDAS}

La información sociodemográfica y de la situación de cuidado se obtuvo a través de un cuestionario autoadministrado elaborado ad hoc que incluía ítems sobre edad, clase social, nivel de estudios, actividad principal, familiar que cuida, diagnóstico, edad y sexo de la persona cuidada, horas diarias dedicadas al cuidado y tiempo cuidando (en años).

La realización del diagnóstico de episodio depresivo mayor actual o pasado y otros trastornos del eje I del DSM se hizo con la Entrevista Clínica Estructurada para los Trastornos del Eje I del DSM-IV, Versión Clínica (Structured Clinical Interview for DSMIV - Clinical Version [SCID-CV]; First, Spitzer, Gibbon y Williams, 1997/1999). Es una entrevista semiestructurada que proporciona diagnósticos del DSM-IV y debe ser administrada por un clínico. Está compuesta por 6 módulos: episodios de estado de ánimo, síntomas psicóticos, trastornos psicóticos, trastornos del estado de ánimo, trastornos por uso de sustancias, ansiedad y otros trastornos. La fiabilidad entre entrevistadores (kappa) es de 61 .

Los síntomas depresivos de las cuidadoras fueron evaluados con la CES-D (Radloff, 1977; versión española de Vázquez et al., 2007), la cual comprende 20 ítems con cuatro opciones de respuesta ( 0 raramente 0 ninguna vez a 3 la mayor parte del tiempo). Su rango de puntuación total oscila entre 0 y 60 , donde puntuaciones más altas indican síntomas depresivos más severos. La consistencia interna de esta escala es de .89.

El malestar emocional fue evaluado con el Cuestionario de Salud General de Goldberg (General Health Questionnaire [GHQ-28]; Goldberg y Hillier, 1979; versión española de Lobo, Pérez-Echeverría y Artal, 1986), el cual contiene 28 ítems divididos en cuatro subescalas (síntomas somáticos, ansiedad e insomnio, disfunción social y depresión grave) con cuatro opciones de respuesta. El rango de puntuación total oscila entre 0 y 28 , donde una mayor puntuación es indicativa de un mayor malestar. La consistencia interna de este cuestionario es de .97 (Godoy-lzquierdo, Godoy, López-Torrecillas y Sánchez-Barrera, 2002).
La sobrecarga de las cuidadoras fue evaluada con la Entrevista de sobrecarga del cuidador (Caregiver Burden Interview [CBI]; Zarit, Reever y Bach-Peterson, 1980), que tiene 22 ítems evaluados en una escala tipo Likert con cinco opciones de respuesta que van de 0 (nunca) a 4 (casi siempre). El rango de la puntuación total va de 0 a 88, existiendo una relación directa entre la puntuación y la sobrecarga experimentada. Su consistencia interna es de .82. Este instrumento fue traducido al español siguiendo las directrices de Guillemin, Bombardier y Beaton (1993), incluyendo el método de traducción-retrotraducción.

\section{- INTERVENCIONES}

a) Intervención cognitivo-conductual: La intervención cognitivo-conductual se basó en el modelo integrador multifactorial de la depresión de Lewinsohn, Hoberman, Teri y Hautzinger (1985). La intervención constó de cinco sesiones de 90 minutos de duración. En la primera sesión se explicó el concepto de depresión y se entrenó a las participantes en la respiración diafragmática. En la segunda sesión se enseñó cómo afectan las actividades al estado de ánimo y se centró en el incremento de las actividades agradables. En la tercera sesión se trató cómo afectan los pensamientos al estado de ánimo, se entrenó a las participantes para cambiar los pensamientos depresivos y se introdujo el autorrefuerzo. La cuarta sesión se dedicó a cómo los contactos con otras personas afectan al estado de ánimo y al incremento de los contactos sociales. Y la quinta sesión se centró en el repaso de lo aprendido y la prevención de recaídas.

b) Grupo control de atención habitual: Las participantes de este grupo no recibieron ninguna intervención psicológica o psicofarmacológica para sus síntomas depresivos así como ningún material psicoeducativo. Sin embargo, se les ofreció la posibilidad de acceder a cualquier servicio médico, psicológico o de servicios sociales disponibles en su comunidad en centros públicos o privados. Los participantes en este grupo fueron evaluados en grupos de aproximadamente cinco cuidadoras en los mismos tiempos que el grupo de intervención. 


\section{- PROCEDIMIENTO}

De forma previa a la intervención se desarrolló y manualizó un protocolo de intervención. A continuación, se llevó a cabo un estudio piloto en el que participaron 10 sujetos y se estudiaron cuestiones específicas relacionadas con el diseño, la aplicación y la viabilidad de la intervención.

Una vez analizada la experiencia piloto e introducidas las modificaciones pertinentes, se llevó a cabo el reclutamiento de la muestra para el ensayo controlado aleatorizado. Todas las cuidadoras fueron evaluadas con anterioridad al inicio de la intervención con el objeto de garantizar el cumplimiento de los criterios de elegibilidad y establecer una línea base. Aquellas que fueron susceptibles de ser elegidas para el estudio, fueron invitadas a participar y asignadas aleatoriamente a la intervención cognitivo-conductual o al grupo control de atención habitual.

Posteriormente, se aplicó la intervención durante cinco sesiones de 90 minutos en grupos de aproximadamente cinco cuidadoras. Tres psicólogas con experiencia clínica entre dos y seis años que habían recibido 35 horas de entrenamiento teórico-práctico en la aplicación de la intervención, implementaron las intervenciones en centros próximos a los domicilios de las participantes. Dos clínicos con más de 17 años de experiencia realizaron el entrenamiento, observaron la grabación de las sesiones y supervisaron semanalmente a las terapeutas. No hubo diferencias significativas entre las terapeutas en los resultados de la intervención respecto a la incidencia de depresión $\left(x^{2}(2, N=170)=1.27, p=.61\right)$, síntomas depresivos $\left(x^{2}(2, N=170)=3.23\right.$, $p=.20)$, malestar emocional $\left(x^{2}(2, N=170)\right.$ $=4.13, p=.12)$ o sobrecarga del cuidador $\left(x^{2}\right.$ $(2, N=170)=4.60, p=.10)$. Se grabaron las sesiones para evaluar el grado de adherencia de las terapeutas al protocolo establecido. La adherencia al protocolo fue del $97 \%$.

Una vez finalizada la intervención, entrevistadores entrenados que no formaban parte del personal investigador y que desconocían las condiciones a las que cada participante había sido asignada realizaron las evaluaciones al final del tratamiento, y a 1, 3, 6, y 12 meses de seguimiento.
- ESTRATEGIA DE ANÁLISIS DE DATOS

Los análisis estadísticos se realizaron con el paquete estadístico SPSS para Windows (versión 20.0). Los análisis se realizaron de acuerdo con el principio de intención de tratar. Los valores perdidos de los sujetos que abandonaron el estudio fueron imputados mediante imputación múltiple con el Programa Amelia II (Honaker, King y Blackwell, 2009).

Se describieron las características demográficas, de cuidado y clínicas de la muestra utilizando tablas de frecuencias y estadísticos descriptivos, y se compararon utilizando la prueba ji cuadrado para las variables discretas (o test exacto de Fisher con valores esperados menores de 5) y pruebas to $F$ para las variables ordinales o continuas.

El porcentaje de participantes que tuvieron un cambio clínicamente significativo en la sintomatología depresiva evaluada por la CES-D fue el resultado principal, definido como presentar recuperación y mostrar mejoría significativa en la CES-D de acuerdo con Jacobson, Follete y Revenstorf (1984) y usando las fórmulas corregidas por Evans, Margison y Barkham (1998). La recuperación fue definida como tener una puntuación $<17$ en la CES-D, de acuerdo a la fórmula corregida (Mclin * DTnorm) + (Mnorm * DTclin)/ DTnorm + DTclin, donde Mclin y DTclin = media y desviación típica de la muestra clínica, respectivamente y Mnorm y DTnorm = media y desviación típica de la población normativa. La mejoría significativa fue determinada calculando el Índice de Cambio Fiable (Evans, 1998), usando la fórmula corregida basada en el cambio que ocurriría menos del $5 \%$ de las veces debido únicamente a la falta de fiabilidad de la medida, i.e., $1.96 * \mathrm{DT} 1 * \sqrt{ } 2 * \sqrt{ }(1-$ rel $)$, donde DT $1=$ desviación típica en línea base y rel $=$ fiabilidad de la medida. Las participantes que tuvieron un cambio clínicamente significativo se codificaron como 1 ('éxito') y los otros como 0 ('fracaso'). Para comparar ambas intervenciones en la posintervención y en los seguimientos a 1, 3, 6 y 12 meses se utilizó la prueba ji cuadrado.

Además, se evaluaron los predictores del cambio clínicamente significativo en la posintervención y en el seguimiento a los 12 meses. Se realizaron análisis de regresión 
logística univariados para cada una de las variables sociodemográficas, de cuidado y clínicas evaluadas en la preintervención. Las variables con una $p \leq .30$ en el análisis exploratorio se incorporaron a un análisis de regresión logística multivariado. Cada modelo, corregido por la sintomatología depresiva preintervención, examinó los efectos principales de esas variables preintervención candidatas sobre la variable resultado en la posintervención y en el seguimiento a 12 meses. Aquellos que produjeron un efecto principal significativo de la variable candidata sobre el resultado indicaban un efecto predictivo.

\section{RESULTADOS}

\section{- CARACTERÍSTICAS DE LA MUESTRA}

La Tabla 1 recoge las características sociodemográficas, de la situación de cuidado y clínicas en la preintervención para la muestra global y cada una de las condiciones experimentales.

En relación a la muestra total $(N=170)$, la media de edad fue de 55.1 años $(D T=9.0)$, el $57.1 \%$ pertenecían a una clase social baja o media-baja, 61.8\% tenía estudios primarios y el $75.9 \%$ se dedicaba a las tareas domésticas. Respecto a las personas cuidadas, el $49.4 \%$ era el padre o la madre de la cuidadora, el $49.4 \%$ tenía demencia, su media de edad eran los 80.4 años $(D T=17.7)$ y el $73.5 \%$ era mujer.

Las cuidadoras dedicaron una media de 17 horas diarias al cuidado durante los últimos 10.1 años. La puntuación media de síntomas depresivos en la preintervención fue de 23.3 $(D T=6.4)$, la media de malestar emocional fue $5.9(D T=4.4)$ y la media de sobrecarga fue de $26.8(D T=12.4)$.

El proceso de asignación al azar produjo grupos comparables, ya que no hubo diferencias significativas entre los dos grupos en ninguna variable.

El porcentaje de abandono fue de $2.3 \%$ $(n=2)$ en el grupo de intervención y $3.7 \%$ $(n=3)$ en el grupo control; el Test exacto de Fisher no mostró diferencias significativas en los abandonos entre ambos grupos $(p=0.677)$.
Tabla 7

Características sociodemográficas, de la situación de cuidado y clínicas de las participantes

\begin{tabular}{|c|c|c|c|c|c|c|}
\hline \multirow[t]{2}{*}{ Variables } & \multicolumn{2}{|c|}{ Total } & \multicolumn{2}{|c|}{$\begin{array}{l}\text { Intervención } \\
\text { Cognitivo- } \\
\text { conductual }\end{array}$} & \multicolumn{2}{|c|}{ Grupo Control } \\
\hline & $N=170$ & $\%$ & $N=88$ & $\%$ & $N=82$ & $\%$ \\
\hline \multicolumn{7}{|l|}{ Edad } \\
\hline$M$ & 55.1 & & 55.7 & & 54.5 & \\
\hline DT & 9.0 & & 9.7 & & 8.2 & \\
\hline \multicolumn{7}{|l|}{ Clase social } \\
\hline Baja/media-baja & 97 & 57.1 & 52 & 57.1 & 45 & 57.1 \\
\hline Media/media-alta & 73 & 42.9 & 36 & 42.9 & 37 & 42.9 \\
\hline \multicolumn{7}{|l|}{$\begin{array}{l}\text { Nivel de } \\
\text { estudios }\end{array}$} \\
\hline $\begin{array}{l}\text { Sabe leer y } \\
\text { escribir }\end{array}$ & 44 & 25.8 & 25 & 25.8 & 19 & 25.8 \\
\hline Primarios & 105 & 61.8 & 52 & 61.8 & 53 & 61.8 \\
\hline $\begin{array}{l}\text { Secundarios/ } \\
\text { universitarios }\end{array}$ & 21 & 12.4 & 11 & 12.4 & 10 & 12.4 \\
\hline \multicolumn{7}{|l|}{$\begin{array}{l}\text { Actividad } \\
\text { principal }\end{array}$} \\
\hline $\begin{array}{l}\text { Tareas } \\
\text { domésticas }\end{array}$ & 129 & 75.9 & 66 & 75.9 & 63 & 75.9 \\
\hline Otras & 41 & 24.1 & 22 & 24.1 & 19 & 24.1 \\
\hline \multicolumn{7}{|l|}{$\begin{array}{l}\text { Familiar } \\
\text { cuidado }\end{array}$} \\
\hline Pareja & 19 & 11.2 & 12 & 11.2 & 7 & 11.2 \\
\hline $\mathrm{Hijo/a}$ & 16 & 9.4 & 13 & 9.4 & 3 & 9.4 \\
\hline Padre/madre & 84 & 49.4 & 40 & 49.4 & 44 & 49.4 \\
\hline Otros familiares & 51 & 30.0 & 23 & 30.0 & 28 & 30.0 \\
\hline \multicolumn{7}{|l|}{$\begin{array}{l}\text { Diagnóstico } \\
\text { de la persona } \\
\text { cuidada }\end{array}$} \\
\hline Demencia & 84 & 49.4 & 42 & 49.4 & 42 & 49.4 \\
\hline Otro & 86 & 50.6 & 46 & 50.6 & 40 & 50.6 \\
\hline \multicolumn{7}{|l|}{$\begin{array}{l}\text { Edad de } \\
\text { la persona } \\
\text { cuidada }\end{array}$} \\
\hline M & 80.4 & & 80.4 & & 80.4 & \\
\hline DT & 17.7 & & 17.8 & & 17.7 & \\
\hline \multicolumn{7}{|l|}{$\begin{array}{l}\text { Sexo de la } \\
\text { persona } \\
\text { cuidada }\end{array}$} \\
\hline Hombre & 45 & 26.5 & 26 & & 19 & \\
\hline Mujer & 125 & 73.5 & 62 & & 63 & \\
\hline \multicolumn{7}{|l|}{$\begin{array}{l}\text { Horas diarias } \\
\text { dedicadas al } \\
\text { cuidado }\end{array}$} \\
\hline M & 17.0 & & 16.8 & & 17.3 & \\
\hline DT & 2.9 & & 3.5 & & 2.2 & \\
\hline
\end{tabular}


Tabla 7

Características sociodemográficas, de la situación de cuidado y clínicas de las participantes (Continuación)

\begin{tabular}{|c|c|c|c|c|c|c|}
\hline \multirow{2}{*}{ Variables } & \multicolumn{2}{|c|}{ Total } & \multicolumn{2}{|c|}{$\begin{array}{l}\text { Intervención } \\
\text { Cognitivo- } \\
\text { conductual }\end{array}$} & \multicolumn{2}{|c|}{ Grupo Control } \\
\hline & $N=170$ & $\%$ & $N=88$ & $\%$ & $N=82$ & $\%$ \\
\hline \multicolumn{7}{|l|}{$\begin{array}{l}\text { Tiempo } \\
\text { cuidando } \\
\text { (años) }\end{array}$} \\
\hline M & 10.1 & & 9.8 & & 10.5 & \\
\hline DT & 6.9 & & 6.9 & & 7.0 & \\
\hline \multicolumn{7}{|l|}{$\begin{array}{l}\text { Síntomas } \\
\text { depresivos }\end{array}$} \\
\hline M & 23.3 & & 23.7 & & 22.8 & \\
\hline DT & 6.4 & & 6.4 & & 6.5 & \\
\hline \multicolumn{7}{|l|}{$\begin{array}{l}\text { Malestar } \\
\text { emocional }\end{array}$} \\
\hline M & 5.9 & & 5.6 & & 6.1 & \\
\hline DT & 4.4 & & 4.3 & & 4.6 & \\
\hline \multicolumn{7}{|c|}{$\begin{array}{l}\text { Sobrecarga } \\
\text { del cuidador }\end{array}$} \\
\hline M & 26.8 & & 26.6 & & 27.0 & \\
\hline DT & 12.4 & & 11.9 & & 12.9 & \\
\hline
\end{tabular}

\section{- CAMBIO CLÍNICAMENTE SIGNIFICATIVO}

En la Tabla 2 se muestran los porcentajes de sujetos que alcanzaron un cambio clínicamente significativo en la sintomatología depresiva en cada grupo. En la posintervención, el $62.5 \%$ ( $n=55)$ de las participantes del grupo de intervención alcanzó un cambio clínicamente significativo, mientras que en el grupo control sólo lo obtuvo el $15.9 \%(n=13)$ de las cuidadoras. La diferencia entre ambos grupos fue estadísticamente significativa $\left(x^{2}\right)(1$, $N=170)=38.48, p<.001)$. Además, estos efectos se mantuvieron a largo plazo. Así, en el seguimiento a 1 mes, un $69.3 \%(n=61)$ de cuidadoras del grupo de intervención obtuvieron un cambio clínicamente significativo, frente al $18.3 \%(n=15)$ del grupo control, siendo estas diferencias estadísticamente significativas $(x 2(1, N=170)=44.71, p<.001)$. En el seguimiento a 3 meses, el $71.6 \%(n=63)$ de las cuidadoras del grupo de intervención tuvieron un cambio clínicamente significativo en comparación con el 19.5\% $(n=16)$ del grupo control; la diferencia entre ambos grupos resultó estadísticamente significativa $\left(x^{2}(1, N=170)\right.$ $=46.28, p<.001)$. En el seguimiento a 6 meses, un $71.6 \%(n=63)$ de cuidadoras del grupo de intervención mostraron un cambio clínico, mientras que esto sólo ocurrió en el 18.3\% $(n=15)$ de las cuidadoras del grupo control. Esta diferencia fue significativa $\left(x^{2}(1, N=170)=48.56, p<.001\right)$. Finalmente, en el seguimiento a 12 meses el $81.8 \%$ $(n=72)$ de las cuidadoras del grupo de intervención alcanzó un cambio clínicamente significativo en comparación con el $17.1 \%(n=14)$ del grupo control, siendo esta diferencia estadísticamente significativa $\left(x^{2}(1, N=170)=71.18, p<.001\right)$.

Tabla 2

Porcentaje de participantes que mostraron cambio clínico significativo en la sintomatología depresiva

\begin{tabular}{|c|c|c|c|c|c|c|}
\hline \multicolumn{7}{|c|}{ Grupos } \\
\hline \multirow[t]{2}{*}{ Evaluaciones } & \multicolumn{2}{|c|}{$\begin{array}{l}\text { Intervención } \\
\text { cognitivo- } \\
\text { conductual } \\
(n=88)\end{array}$} & \multicolumn{2}{|c|}{$\begin{array}{c}\text { Grupo control } \\
(n=82)\end{array}$} & \multirow[b]{2}{*}{$x^{2}$} & \multirow[b]{2}{*}{$p$} \\
\hline & $n$ & $\%$ & $n$ & $\%$ & & \\
\hline \multicolumn{7}{|l|}{ Posintervención } \\
\hline $\begin{array}{l}\text { Sin cambio clínicamente } \\
\text { significativo }\end{array}$ & 33 & 37.5 & 69 & 84.1 & & \\
\hline $\begin{array}{l}\text { Con cambio clínicamente } \\
\text { significativo }\end{array}$ & 55 & 62.5 & 13 & 15.9 & 38.48 & $<.001$ \\
\hline \multicolumn{7}{|l|}{ Seguimiento a 1 mes } \\
\hline $\begin{array}{l}\text { Sin cambio clínicamente } \\
\text { significativo }\end{array}$ & 27 & 30.7 & 67 & 81.7 & & \\
\hline $\begin{array}{l}\text { Con cambio clínicamente } \\
\text { significativo }\end{array}$ & 61 & 69.3 & 15 & 18.3 & 44.71 & $<.001$ \\
\hline \multicolumn{7}{|l|}{$\begin{array}{l}\text { Seguimiento a } 3 \\
\text { meses }\end{array}$} \\
\hline $\begin{array}{l}\text { Sin cambio clínicamente } \\
\text { significativo }\end{array}$ & 25 & 28.4 & 66 & 80.5 & & \\
\hline $\begin{array}{l}\text { Con cambio clínicamente } \\
\text { significativo }\end{array}$ & 63 & 71.6 & 16 & 19.5 & 46.28 & $<.001$ \\
\hline \multicolumn{7}{|l|}{$\begin{array}{l}\text { Seguimiento a } 6 \\
\text { meses }\end{array}$} \\
\hline $\begin{array}{l}\text { Sin cambio clínicamente } \\
\text { significativo }\end{array}$ & 25 & 28.4 & 67 & 81.7 & & \\
\hline $\begin{array}{l}\text { Con cambio clínicamente } \\
\text { significativo }\end{array}$ & 63 & 71.6 & 15 & 18.3 & 48.56 & $<.001$ \\
\hline \multicolumn{7}{|l|}{$\begin{array}{l}\text { Seguimiento a } 12 \\
\text { meses }\end{array}$} \\
\hline $\begin{array}{l}\text { Sin cambio clínicamente } \\
\text { significativo }\end{array}$ & 16 & 18.2 & 68 & 82.9 & & \\
\hline $\begin{array}{l}\text { Con cambio } \\
\text { clínicamente significativo }\end{array}$ & 72 & 81.8 & 14 & 17.1 & 71.18 & $<.001$ \\
\hline
\end{tabular}

\section{- VARIABLES PREDICTORAS DEL CAMBIO CLIINICAMENTE RELEVANTE}

En la Tabla 3 se recogen los resultados exploratorios de los análisis de regresión logística univariados del cambio clínicamente significativo en el grupo de intervención para 
la posintervención y el seguimiento a 12 meses. Las variables halladas como potenciales predictoras $(p \leq .30)$ en la posintervención fueron la clase social, la actividad principal, el familiar cuidado, la edad de la persona cuidada y el malestar emocional en la línea base; mientras que las halladas como potenciales predictoras en el seguimiento a 12 meses fueron la edad de la cuidadora, la actividad principal, el familiar cuidado, la edad de la persona cuidada, el sexo de la persona cuidada, la sintomatología depresiva y el malestar emocional en la preintervención. Estas variables fueron incluidas en los correspondientes análisis multivariantes.

En la Tabla 4 se recogen los predictores del cambio clínicamente significativo en la posintervención en el análisis multivariante.

Las variables predictoras del cambio clínicamente significativo en la posintervención fueron tener como actividad principal las tareas domésticas $(O R=7.35,1 C 95 \%, 1.35-40.13, p$ $=.021)$ y una mayor edad de la persona cuidada $(O R=1.04, I C 95 \%, 1.01-1.08, p=.043) . \mathrm{En}$ el seguimiento a 12 meses, sin embargo, no se halló ninguna variable predictora del cambio clínicamente significativo.

Análisis exploratorios: resultados de los análisis de regresión logística univariados para el grupo de intervención cognitivo-conductual

\begin{tabular}{|c|c|c|c|c|}
\hline \multirow{2}{*}{ Predictor potencial } & \multicolumn{2}{|c|}{ Posintervención } & \multicolumn{2}{|c|}{ Seguimiento a 12 meses } \\
\hline & OR (IC 95\%) & $p$ & OR (IC 95\%) & $p$ \\
\hline Edad & $1.00(0.96-1.05)$ & .866 & $0.96(0.91-1.02)$ & .185 \\
\hline \multicolumn{5}{|l|}{ Clase social } \\
\hline Baja/media-baja & $1.65(0.67-3.96)$ & .264 & $0.60(0.19-1.91)$ & .388 \\
\hline Media/media-alta & 1 (ref) & & 1 (ref) & \\
\hline \multicolumn{5}{|l|}{ Nivel educativo } \\
\hline Sabe leer y escribir & $1.02(0.23-4.44)$ & .983 & $0.70(012-4.20)$ & .700 \\
\hline Primarios & $0.91(0.24-3.53)$ & .896 & $1.22(0.22-6.74)$ & .818 \\
\hline Secundarios/universitarios & 1 (ref) & & 1 (ref) & \\
\hline \multicolumn{5}{|l|}{ Actividad principal } \\
\hline Tareas domésticas & $5.28(1.42-19.57)$ & .013 & $2.10(0.66-6.66)$ & .208 \\
\hline Otras & 1 (ref) & & 1 (ref) & \\
\hline \multicolumn{5}{|l|}{ Familiar cuidado } \\
\hline Pareja & $0.15(0.29-0.78)$ & .025 & $1.06(0.21-5.27)$ & .944 \\
\hline $\mathrm{Hijo/a}$ & $0.67(0.12-3.62)$ & .002 & $1.18(0.24-5.77)$ & .841 \\
\hline Padre/madre & $0.25(0.63-0.99)$ & .048 & $3.18(0.79-12.76)$ & .103 \\
\hline Otros familiares & 1 (ref) & & 1 (ref) & \\
\hline \multicolumn{5}{|l|}{ Diagnóstico de la persona cuidada } \\
\hline Demencia & $1.41(0.59-3-36)$ & .441 & $1.67(0.55-5.07)$ & .368 \\
\hline Otro & 1 (ref) & & 1 (ref) & \\
\hline Edad de la persona cuidada & $1.04(1.01-1.07)$ & .010 & $1.02(0.99-1.05)$ & .146 \\
\hline \multicolumn{5}{|l|}{ Sexo de la persona cuidada } \\
\hline Mujer & $1.33(0.52-3.40)$ & .547 & $2.17(0.71-6.64)$ & .175 \\
\hline Hombre & 1 (ref) & & 1 (Ref) & \\
\hline Horas diarias dedicadas al cuidado & $0.94(0.81-1.08)$ & .349 & $0.97(0.81-1.15)$ & .700 \\
\hline Tiempo cuidando (años) & $1.01(0.95-1.08)$ & .751 & $0.96(0.90-1.04)$ & .310 \\
\hline Síntomas depresivos & $1.03(0.96-1.11)$ & .346 & $1.07(0.97-1.18)$ & .166 \\
\hline Malestar emocional & $0.95(0.86-1.05)$ & .304 & $1.08(0.94-1.24)$ & .270 \\
\hline Sobrecarga del cuidador & $1.01(0.97-1.04)$ & .724 & $1.01(0.97-1.06)$ & .672 \\
\hline
\end{tabular}


Tabla 4

Resultados de los análisis de regresión logística multivariados para la predicción de cambio clínicamente significativo de los síntomas depresivos posintervención para el grupo de intervención cognitivoconductual

\begin{tabular}{|c|c|c|}
\hline \multirow{2}{*}{ Predictor } & \multicolumn{2}{|c|}{ Posintervención } \\
\hline & OR (IC 95\%) & $p$ \\
\hline \multicolumn{3}{|l|}{ Clase social } \\
\hline Baja/media-baja & $1.21(0.41-3.58)$ & .726 \\
\hline Media/media-alta & 1 (Ref) & \\
\hline \multicolumn{3}{|l|}{$\begin{array}{l}\text { Actividad } \\
\text { principal }\end{array}$} \\
\hline Tareas domésticas & $7.35(1.35-40.13)$ & .021 \\
\hline Otras & 1 (Ref) & \\
\hline \multicolumn{3}{|l|}{ Familiar cuidado } \\
\hline Pareja & $0.26(0.36-1.92)$ & .189 \\
\hline $\mathrm{Hijo/a}$ & $0.95(0.19-4.67)$ & .948 \\
\hline Padre/madre & $2.33(0.33-16.30)$ & .394 \\
\hline Otros familiares & $1(\operatorname{Ref})$ & \\
\hline $\begin{array}{l}\text { Edad de la } \\
\text { persona cuidada }\end{array}$ & $1.04(1.01-1.08)$ & .043 \\
\hline $\begin{array}{l}\text { Malestar } \\
\text { emocional }\end{array}$ & $1.05(0.96-1.15)$ & .254 \\
\hline
\end{tabular}

\section{DISCUSIÓN}

Los principales objetivos de este estudio fueron determinar el cambio clínicamente significativo en los síntomas depresivos en un estudio de prevención indicada de la depresión con cuidadoras que participaron en una intervención cognitivo-conductual de prevención indicada de la depresión, así como estudiar las variables predictoras del cambio clínico tras la intervención y el seguimiento a 12 meses.

Al final de la intervención, se encontró un porcentaje significativamente mayor de cuidadoras que alcanzaron un cambio clínicamente significativo en el grupo de intervención en comparación con el grupo control $(62.5 \%$ vs. $15.9 \%, p<.001)$. Y esto se mantuvo hasta los 12 meses de seguimiento, donde un $81.8 \%$ de cuidadoras obtuvieron un cambio clínico en el grupo de intervención frente al $17.1 \%$ del grupo control $(p<.001)$. Estos datos indican que la mayoría de las cuidadoras tratadas logró recuperar su nivel de funcionalidad al nivel de la población sin síntomas depresivos, y en mayor medida que el grupo control.

Este hallazgo en la posintervención para el grupo de intervención fue menor al $80.9 \%$ de cuidadoras con significación clínica encontrado en el estudio de Vázquez et al. (2013); si bien estas diferencias pueden deberse al distinto procedimiento para considerar el cambio clínico, que en el presente estudio fue más restrictivo en comparación con el punto de corte empleado en el estudio de Vázquez et al. (2013). Además, el presente resultado fue similar al $62.9 \%$ encontrado en el estudio de Vázquez et al. (2012) con estudiantes universitarios, y superior al $48 \%$ informado por Liv et al. (2009) en adultos.

Respecto al impacto de la intervención a los 12 meses de seguimiento, nuestro hallazgo fue superior al $70.8 \%$ de cuidadoras con mejoría significativa encontrado en el estudio de Otero, Smit et al. (2015) en el mismo momento de evaluación, así como al $43 \%$ de adolescentes a los 6 meses en el estudio de Stice et al. (2008) y al $31.5 \%$ a los 2 años de seguimiento (Stice et al., 2010). Sin embargo, fue inferior al $95 \%$ encontrado por Konnert et al. (2009) a los 6 meses de seguimiento. Una posible explicación a esta divergencia es el procedimiento diferente empleado en el estudio de Konnert et al. (2009) para calcular la significación clínica (basado en la comparación con la puntuación de la muestra normativa), así como posibles sesgos debidos al pequeño tamaño muestral de este estudio.

El análisis de las variables predictoras del cambio clínicamente significativo encontró que tras la intervención hubo un mayor cambio clínico en aquellas cuidadoras que se dedicaban a las tareas domésticas y que cuidaban familiares de mayor edad. El hallazgo de que las amas de casa obtuvieran mejores resultados con la intervención es prometedor, dado que se ha encontrado de forma consistente que la mayoría (73.1\%) de los cuidadores no tienen empleo (Instituto de Mayores y Servicios Sociales, 2005) y tienen un 50\% más de probabilidades de ser amas de casa que los no cuidadores (Colombo, Llena-Nozal, Mercier y Tiadens, 2011). Una posible explicación para este hallazgo es que las cuidadoras que no trabajan fuera de casa están menos expuestas a la variedad de estresores derivados 
de compaginar el trabajo con el cuidado de un familiar dependiente (Scharlach, Sobel y Roberts, 1991) y tienen más disponibilidad de tiempo para dedicar a la intervención y realizar las tareas para casa, estando esto último relacionado con la obtención de mejores resultados tras la intervención (Otero, Vázquez et al., 2015). Futuros estudios podrían diseñar programas más breves o con formatos no presenciales para aquellos cuidadores que compatibilizan el trabajo con el cuidado de un familiar en situación de dependencia.

Por otra parte, el hallazgo de que cuidar de un familiar de mayor edad fue predictor del cambio clínico en la posintervención carece, hasta donde alcanza nuestro conocimiento, de antecedentes en la literatura científica. Una explicación tentativa de este resultado está relacionada con el duelo anticipado, sugiriendo que las cuidadoras aceptan mejor la pérdida progresiva de su familiar si es una persona mayor que si es un niño o joven (Centeno, 2013), lo que contribuye a presentar menos síntomas depresivos tras la intervención. Es necesaria más investigación sobre la relación entre estas variables. Las restantes variables analizadas en la línea base (clase social de la cuidadora, familiar cuidado y malestar emocional) no fueron predictoras del cambio clínico en la posintervención cuando se analizaron simultáneamente.

En la misma línea, a los 12 meses de seguimiento no se encontró ninguna variable predictora del cambio clínicamente significativo. Esto indica que la intervención fue igualmente efectiva para todo tipo de participantes. Algunos factores que pudieron contribuir a este alto grado de generalización de los resultados fueron la realización de un estudio piloto (que permitió adecuar el contenido de la intervención y los materiales utilizados a esta población), así como el desarrollo de una intervención basada en un modelo teórico, la selección de las variables de resultado apropiadas y la selección de la muestra en función del nivel de sintomatología depresiva, en línea con las recomendaciones de Zarit y Femia (2008).

Este trabajo debe ser interpretado teniendo en cuenta sus limitaciones. Nuestra muestra sólo incluyó mujeres, por lo que desconocemos si el sexo del cuidador podría ser un predictor del cambio clínico en la sintomatología depresiva. Aunque esta limitación es mínima en la práctica clínica, debido a que la mayoría de los cuidadores son mujeres (Colombo et al., 2011; Instituto de Mayores y Servicios Sociales, 2005). Además, existen variables que no han sido exploradas y que podrían ser predictoras de los resultados de la intervención relacionadas tanto con características de los cuidadores (e.g., autoeficacia, resiliencia, personalidad), como con la adherencia al tratamiento (e.g., número de sesiones asistidas o de tareas para casa realizadas). Futuros estudios podrían analizar estas variables.

A pesar de estas limitaciones, este estudio presenta importantes aportaciones para la investigación y la práctica clínica. Proporciona evidencia de la significación clínica de los efectos de la intervención sobre la sintomatología depresiva y de las variables predictoras del resultado, lo cual es de gran interés para conocer el impacto clínico de la intervención y la optimización de la misma. A diferencia de la mayoría de los estudios que se basan en pruebas de significación estadística (que sólo tienen en cuenta la media de la muestra), este estudio proporciona información valiosa sobre la variabilidad de los efectos de la intervención para los diferentes individuos dentro de la muestra. Además, conocer no sólo la eficacia de la intervención sino también cuál es su alcance en la vida de las personas y para quién resulta más eficaz contribuye al diseño de tratamientos más personalizados y efectivos.

\section{- Conflicto de intereses}

Los autores declaran no tener ningún conflicto de intereses.

- Agradecimientos: El presente trabajo forma parte de una investigación financiada por el proyecto 2007/PN017 del Ministerio de Trabajo y Asuntos Sociales de España. Agradecemos el apoyo de la Secretaría Xeral de Política Social, Unidad Técnica de Coordinación de Dependencia, Consellería de Trabajo y Bienestar de la Xunta de Galicia.

\section{REFERENCIAS}

Centeno, C. (2013). Gestión del duelo y las pérdidas. Aprendiendo a convivir con lo ausente. Jaén: Formación Alcalá

Colombo, F., Llena-Nozal., A., Mercier, J. y Tjadens, F. (2011). Help Wanted? Providing and 
Paying for Long-term Care. París: OECD Publishing, OECD Health Policy Studies.

Cuiipers, P., Smit, F. y Willemse, G. (2005). Predicting the onset of major depression in subjects with subthreshold depression in primary care: A prospective study. Acta Psychiatrica Scandinavica, 177, 133-138.

http://dx.doi.org/10.1111/i.1600$\underline{0447.2004 .00416 . x}$

Dobkin, R. D., Rubino, J. T., Allen, L. A., Friedman, J., Gara, M. A., Mark, M. H. y Menza, M. (2012). Predictors oftreatment response to cognitive-behavioral therapy for depression in Parkinson's disease. Journal of Consulting and Clinical Psychology, 80, 694-699. http://dx.doi.org/10.1037/a0027695

Evans, C. (1998). Reliable Change Criterion Calculation. Recuperado de http://www.psyctc.org/ stats/rcscl.htm

Evans, C., Margison, F. y Barkham, M. (1998). The contribution of reliable and clinically significant change methods to evidence-based mental health. Evidence-Based Mental Health, 1, 70-72. http://dx.doi.org/10.1136/ebmh.1.3.70

First, M. B., Spitzer, R. L., Gibbon, M. y Williams, J. B. W. (1999). Entrevista Clínica Estructurada para los Trastornos del Eje I del DSMIV- Versión Clínica (SCID-CV). Barcelona: Masson. (Original publicado en 1997).

Gabilondo, A., Rojas-Farreras, S., Vilagut, G., Haro, J. M., Fernández, A., Pinto-Meza, A. y Alonso, J. (2010). Epidemiology of major depressive episode in a southern European country: Results from the ESEMeDSpain project. Journal of Affective Disorders 120, 76-85. http://dx.doi.org/10.1016/i.jad.2009.04.016

Godoy-lzquierdo, D., Godoy, J. F., LópezTorrecillas, F. y Sánchez-Barrera, M. B. (2002). Propiedades psicométricas de la versión española del "Cuestionario de salud general de Goldberg-28". Revista de Psicología de la Salud, 14, 49-71.

Goldberg, D. P. y Hillier, V. F. (1979). A scaled version of the General Health Questionnaire. Psychological Medicine, 9, 139-145. http://dx.doi.org/10.1017/s0033291700021644

Grady, D., Cummings, S. R. y Hulley, S. B. (2008). Otros diseños de ensayos clínicos y temas de implementación. En S. B. Hulley, S. M. Cummings, W. S. Browner, D. G. Grady y T. B. Newman (Eds.), Diseño de investigaciones clínicas (pp. 185-206, $3^{a}$ Ed.). Barcelona: Lippincott Williams \& Wilkins. (Original publicado en 2007).

Guillemin, F., Bombardier, C. y Beaton, D. (1993). Cross-cultural adaptation of health-related quality of life measures: Literature review and proposed guidelines. Journal of Clinical Epidemiology, 46, 1417-1432.

http://dx.doi.org/10.1016/0895-4356(93)90142-N

Honaker, J., King, G. y Blackwell, M. (2009). Amelia II: A program for missing data. Recuperado de http://gking.harvard.edu/amelia

Institute of Medicine (1994). Reducing risks for mental disorders: Frontiers for preventive intervention research. Washington, DC: National Academy Press.

Instituto de Mayores y Servicios Sociales (Ed.). (2005). Cuidados a las personas mayores en los hogares españoles. El entorno familiar. Madrid: Ministerio de Trabajo y Asuntos Sociales. Recuperado de http://gking.harvard.edu/amelia

Jacobson, N. S., Follete, W. C. y Revenstorf, D. (1984). Psychotherapy outcome research: Methods of reporting variability and evaluation clinical significance. Behavior Therapy, 15, 336-352.

http://dx.doi.org/10.1016/S0005-7894(84)80002-7 Jacobson, N. S. y Truax, P. (1991). Clinical significance: A statistical approach to defining meaningful change in psychotherapy research. Journal of Consulting and Clinical Psychology, 59, 12-19.

Kazdin, A. E. (1999). The meanings and measurement of clinical significance. Journal of Consulting and Clinical Psychology, 67, 332-339. http://dx.doi.org/10.1037/0022-006X.67.3.332

Konnert, C., Dobson, K. y Stelmach, L. (2009).

The prevention of depression in nursing home residents: A randomized clinical trial of cognitive-behavioral therapy. Aging \& Mental Health, 13, 288-299. http://dx.doi.org/10.1080/13607860802380672

Kraemer, H. C., Wilson, G. T., Fairburn, C. G. y Agras W. S. (2002). Mediators and moderators of treatment effects in randomized clinical trials. Archives of General Psychiatry, 59, 877-883.

http://dx.doi.org/10.1001/archpsyc.59.10.877

Lewinsohn, P. M., Hoberman, H., Teri, L. y Hautzinger, M. (1985). An integrative theory of depression. In S. Reiss y R. R. Bootzin (Eds.), Theoretical issues in behavior therapy (pp. 331 -359). New York: Academic Press.

Liu, E. T. H., Chen, W. L., Li, Y. H., Wang, C. H., Mok, T. J. y Huang, H. S. (2009). Exploring the efficacy of cognitive bibliotherapy and a potential mechanism of change in the treatment of depressive symptoms among the Chinese: A randomized controlled trial. Cognitive Therapy and Research, 33, 449-461.http:// dx.doi.org/10.1007/s10608-008-9228-4

Lobo, A., Perez-Echeverría, M. J. y Artal, J. 
(1986). Validity of the scaled version of the General Health Questionnaire (GHQ-28) in a Spanish population. Psychological Medicine, 16, 135-140. http://dx.doi.org/10.1017/s0033291700002579

Otero, P., Smit, F., Cuiipers, P., Torres, A., Blanco, V. y Vázquez, F. L. (2015). Long-term efficacy of indicated prevention of depression in nonprofessional caregivers: Randomized controlled trial. Psychological Medicine, 45, 1401-1412.

http://dx.doi.org/10.1017/s0033291714002505

Otero, P., Vázquez, F. L., Hermida, E., Díaz, O. y Torres, A. (2015). Relationship of cognitive behavioral therapy effects and homework in an indicated prevention of depression intervention for non-professional caregivers. Psychological Reports, 176, 841-854.

http://dx.doi.org/10.2466/02.PR0.116k22w7

Radloff, L. S. (1977). The CES-D scale: A self-report depression scale for research in the general population. Applied Psychological Measurement, 1, 385-401.

http://dx.doi.org/10.1177/014662167700100306

Scharlach, A. E., Sobel, E. L. y Roberts, R. E. L. (1991). Employment and caregiver strain: An integrative model. Gerontologist, 31, 778-787.

http://dx.doi.org/10.1093/geront/31.6.778

Spek, V., Nyklíček, I., Cuiipers, P. y Pop, V. (2008). Predictors of outcome of group and internetbased cognitive behavior therapy. Journal of Affective Disorders, 105, 137-145.

http://dx.doi.org/10.1016/i.jad.2007.05.001

Stice, E., Rohde, P., Seeley, J. R. y Gau, J. M. (2008). Brief cognitive-behavioral depression prevention program for high-risk adolescents outperforms two alternative interventions: A randomized efficacy trial. Journal of Consulting and Clinical Psychology, 76, 595-606.

http://dx.doi.org/10.1037/a0012645

Stice, E., Rohde, P., Gau, J. M. y Wade, E. (2010). Efficacy trial of a brief cognitive-behavioral depression prevention program for high-risk adolescents: Effects at 1-and 2-year follow-up. Journal of Consulting and Clinical Psychology, 78, 856-867.

http://dx.doi.org/10.1037/a0020544

Torres, Á., Blanco, V., Vázquez, F. L., Díaz, O., Otero, P. y Hermida, E. (2015). Prevalence of major depressive episodes in non-professional caregivers. Psychiatry Research, 226, 333-339.

http://dx.doi.org/10.1016/i.psychres.2014.12.066

Vázquez, F. L., Blanco, V. y López, M. (2007). An adaptation of the Center for Epidemiologic Studies
Depression Scale for use in non-psychiatric Spanish populations. Psychiatry Research, 149, 247-252. http://dx.doi.org/10.1016/i.psychres.2006.03.004

Vázquez, F. L., Blanco, V., Torres, A., Otero, P. y Hermida, E. (2014). La eficacia de la prevención indicada de la depresión: una revisión. Anales de Psicología, 30, 9-24.

http://dx.doi.org/10.6018/analesps.30.1.138931

Vázquez, F. L., Hermida, E., Torres, A., Otero, P., Blanco, V. y Díaz, O. (2014). Eficacia de una intervención preventiva cognitivo-conductual en cuidadoras con síntomas depresivos elevados. Behavioral Psychology/Psicología Conductual, 22, 79-96.

Vázquez, F. L., Otero, P., Torres, A., Hermida, E., Blanco, V. y Díaz, O. (2013). A brief problem-solving indicated-prevention intervention for prevention of depression in nonprofessional caregivers. Psicothema, 25, 87-92.

http://dx.doi.org/10.7334/psicothema2012.89

Vázquez, F. L., Torres, A., Blanco, V., Díaz, O., Otero, P. y Hermida, E. (2012). Comparison of relaxation training with a cognitive-behavioural intervention for indicated prevention of depression in university students: A randomized controlled trial. Journal of Psychiatric Research, 46, 1456-1463.

http://dx.doi.org/10.1016/i.jpsychires.2012.08.007 Vázquez, F. L., Torres, A., Blanco, V., Otero, P., Díaz, O. y Ferraces, M. J. (2016). Long-term followup of a randomized clinical trial assessing the efficacy of a brief cognitive-behavioral depression prevention intervention for caregivers with elevated depressive symptoms. American Journal of Geriatric Psychiatry. Advance online.

http://dx.doi.org/10.1016/i.jagp.2016.02.050

Wang P. S. y Kessler R.C. (2006). Global burden of mood disorders. En D. J. Stein, D. J. Kupfer, y A. F. Schatzberg (Eds.), The American psychiatric publishing textbook of mood disorders (pp. 55-68). Washington, DC: American Psychiatric Publishing, Inc.

Zarit, S. H. y Femia, E. E. (2008). A future for family care and dementia intervention research? Challenges and strategies. Aging \& Mental Health, 12,5-13.

http://dx.doi.org/10.1080/13607860701616317

Zarit, S. H., Reever, K. E. y Bach-Peterson, J. (1980). Relatives of the impaired elderly: Correlates of feelings of burden. Gerontologist, 20, 649-655. http://dx.doi.org/10.1093/geront/20.6.649 